\title{
A near real time system for tropospheric monitoring using IGS hourly data
}

\author{
A. Flores, A. Escudero, M. J. Sedó, and A. Rius \\ Institut d'Estudis Espacials de Catalunya, Edif. Nexus-204, Gran Capità 2-4, 08034 Barcelona, Spain
}

(Received December 27, 1999; Revised June 30, 2000; Accepted July 10, 2000)

\begin{abstract}
The availability of real-time tropospheric data is an important question for atmospheric parameters to be ingested in weather prediction models or in monitoring studies. In the frame of the EU funded project CLIMAP we have developed an on-line system that acquires GPS data from a number of IGS stations distributed around Europe providing hourly batches of GPS observables. An off-line system processing data with a one-day delay was run during one year to tune the characterisitics of the present on-line system. The hourly data are processed using GIPSY/OASIS II software, to obtain the Zenith Total Delay in a near-real-time basis. Every hour, the new batch is merged with the observations of the previous 23 hours to allow the system to process enough data to yield robust results. The question of what prediction for orbits to use has also been addressed, designing the system to always use the best orbits available from the Center for Orbit Determination in Europe, using the quality codes for the prediction of the orbits to remove "bad quality" satellites from the solution. To validate the results, the ZTD time series for each station have been compared against the solutions obtained through a Precise Point Positioning using final precise orbits and clock corrections. The estimation processing has been tuned to account for the particularities of a near-real-time scenario such as the satellite clock correction and the day boundary issue.
\end{abstract}

\section{Introduction}

The Global Positioning System (GPS) provides a source of continuous and global data that can be used to extract tropospheric parameters (see e.g. Bevis et al., 1992; Duan et al., 1996). In particular, the Zenith Total Delay at each station is the parameter estimated in a GPS data processing. This is usually split into the Zenith Hydrostatic Delay (ZHD) and the Zenith Wet Delay (ZWD). The former is mainly driven by the contents of neutral gases of the atmosphere and can be accurately estimated using ground pressure measurements (Elgered et al., 1991); the latter is directly related to the water vapour contents of the troposphere. The spatial and temporal evolution of water vapor is a key variable in the formation and evolution of perturbed weather systems and, thus, the Numerical Weather Prediction Models (NWPM) would be enhanced if they could include these atmospheric data on a near-real-time basis and from a sufficiently dense network in the region considered.

As stated, the derivation of ZWD stems from the extraction of ZTD and the modelisation of ZHD using ground measurements. Hence, if we aim to incorporate such data in a NWPM, we might focus on the obtention of ZTD and let the inherent model separate the wet component.

In this paper we present a system for a near-real-time monitoring of Zenith Total Delay parameter (ZTD) using a network of GPS ground stations distributed around Europe, which has been developed in the frame of the European Union (EU) funded project CLIMAP ${ }^{1}$. There are other

${ }^{1}$ CLimate and environment Monitoring with GPS Atmospheric Profiling.

Copy right(C) The Society of Geomagnetism and Earth, Planetary and Space Sciences (SGEPSS); The Seismological Society of Japan; The Volcanological Society of Japan; The Geodetic Society of Japan; The Japanese Society for Planetary Sciences. prototypes of near-real-time monitoring systems such as the one at $\mathrm{UCAR}^{2}$ or $\mathrm{GFZ}^{3}$ in Europe.

The paper is organized as follows: in the next section we revise the off-line system, which served as a first approach to the Near Real Time (NRT) system, and provided different precision solutions with 1-day, 7 days and 14 days delay. Section 3 is devoted to the NRT system, also called on-line system, since the products are provided on an hourly basis; we describe the processing approach, accuracies of the products and some results. Next, in Section 4, we relate some problems and issues of the on-line system. Finally, some conclusions are presented in Section 5.

\section{The Off-Line System}

In the off-line system data from a set of 27 stations uniformly distributed around Europe (see Fig. 1) are processed using orbit predictions from Center for Orbit Determination in Europe (CODE) located in Berne. GPS data are gathered from two servers mainly: the University of Berne and from the IGS data center in the University of California in San Diego. The set of stations was chosen on the basis of reliability and availability of data, besides their geographical distribution. The incoming data are in Receiver Independent Exchange (RINEX) format and in 24-hours files. A quality check using the TEst of Quality Check (TEQC) software form University NAVSTAR Consortium (UNAVCO) is performed. When a data file fails this quality check, it is then removed from the raw data set to be processed in order to avoid bad quality results. This checking considers the correct format of the RINEX file and the completeness of data that could cause processing errors. Data are processed with

\footnotetext{
${ }^{2}$ http: //www.gst.ucar.edu/gpsrg/realtime.html.

${ }^{3}$ http: //www.gfz.de.
} 


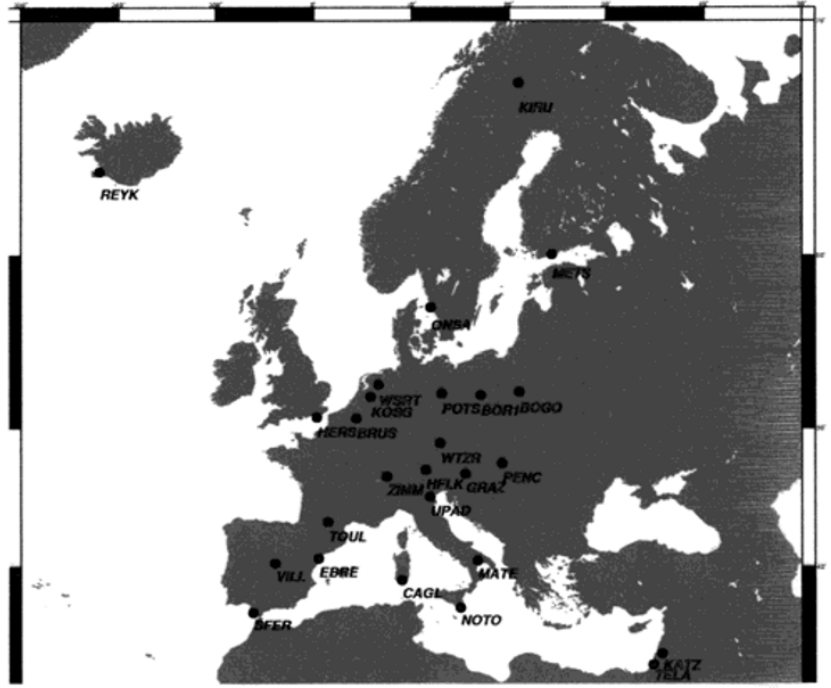

Fig. 1. Location of stations for the off-line system.

GIPSY-OASIS II package software (Webb and Zumberge, 1997). The processing considers a span of one day of data and the predicted orbits, but no clock corrections. Therefore, we constrain the positions of the stations to some millimeters accuracy and estimate the ZTD time series, modelled as a random walk stochastic process, and clock corrections, modelled as white noise stochastic process, and taking a stable station as the reference clock (usually the IGS sites at Onsala or Madrid). The rest of processing parameters are set to follow standard practices, that is, we consider ocean loading effects, antenna phase center corrections, Niell mapping functions (Niell, 1996) and Earth rotation parameters. Regarding orbit predictions, we use the 48 hours predictions from CODE and, based on the quality codes included in the files, we remove from the solution those satellites whose orbits are not reliable (see EC Wavefront Project Final Report 1999). We thus every day provide, as products, the ZTD time series and the clock corrections for the GPS satellites for the previous day.

In order to tune and afterwards to check the validity of the processing approach, the same set of data is processed a posteriori using rapid orbits and clock corrections estimations, provided by the Jet Propulsion Laboratory (JPL) typically within 4 days, and then using the precise fiducial orbits and clock corrections also from JPL, usually 14 days later. When clock corrections were used, only station clock corrections were estimated and the positions of the stations were relaxed to some centimeters: in other words, we have followed a Precise Point Positioning, PPP, (Zumberge et al., 1997); the solutions for the stations locations were then fed back into the system. We thus have three processing strategies in the off-line system, which we name according to the type of orbit estimation used: Predicted Orbits (PO), which is the off-line system as such, Rapid Orbits (RO) and Final Orbits (FO). The complete system was started in March 1998 and has been running since then with different upgrades. The main objective here was to gain experience in the strategy to follow both for the selection of stations and data quality issues and for the processing approach. Regarding the latter,
Table 1. Difference of ZTD time series for a month using solution with precise orbits as reference. Bias $\Delta$ and $\sigma$ are in $\mathrm{cm}$. and in absolute value.

\begin{tabular}{ccccc}
\hline STA & $\Delta$ PO & $\sigma$ PO & $\Delta$ RO & $\sigma$ RO \\
\hline BRUS & 0.104 & 0.214 & 0.036 & 0.149 \\
KIRU & 0.119 & 0.392 & 0.011 & 0.130 \\
ONSA & 0.134 & 0.312 & 0.040 & 0.156 \\
WTZR & 0.086 & 0.224 & 0.036 & 0.162 \\
ZIMM & 0.117 & 0.331 & 0.046 & 0.196 \\
\hline
\end{tabular}

Table 2. Values for accumulated bias and accumulated $\sigma$ using a PPP technique with the clock corrections provided by the off-line system and using the precise solutions from JPL.

\begin{tabular}{ccc}
\hline STA & acc_bias $(\mathrm{cm})$ & acc_o $(\mathrm{cm})$ \\
\hline BRUS & 0.026 & 0.046 \\
KIRU & 0.443 & 0.039 \\
ONSA & 0.026 & 0.045 \\
WTZR & 0.022 & 0.037 \\
ZIMM & 0.026 & 0.048 \\
\hline
\end{tabular}

after some tuning, we have verified the products comparing the three solutions.

On the one hand we took the time series solutions for one month for all the 27 stations (this time span was considered to be sufficient to provide statistically significant results and to guarantee the existance of continuous data for most of the stations, as well as that the automatic system had settled), and for the three processing strategies; we then considered the FO solution as the reference and computed the bias and rms values of the time series solutions of PO and RO. In Table 1 we show the numerical values, in $\mathrm{cm}$, for five of the stations which will later be in the on-line system (described below). As it can be seen, we have achieved sub-centimeter accuracy.

On the other hand, we have verified the validity of the clock corrections for the GPS satellites, which is an output of the off-line system. In order to do that, we have applied the PPP strategy using the precise JPL products and using $\mathrm{PO}$ and the time series of clock corrections, and extracted the ZTD solutions for some stations. In Table 2 we show the accumulated bias and rms of the comparison of both solutions. We clearly see that the Clock Corrections output can be used to perform Point Positioning.

\section{The On-Line System}

\subsection{Description of on-line system}

Once the off-line system was steadily working and we had developed the tools for the automatization of the process as well as having tuned the strategy, we launched the on-line system. For that, we selected a set of seven stations in Europe, covering the whole region. The number was reduced in order to speed up the process since the results should be avail- 


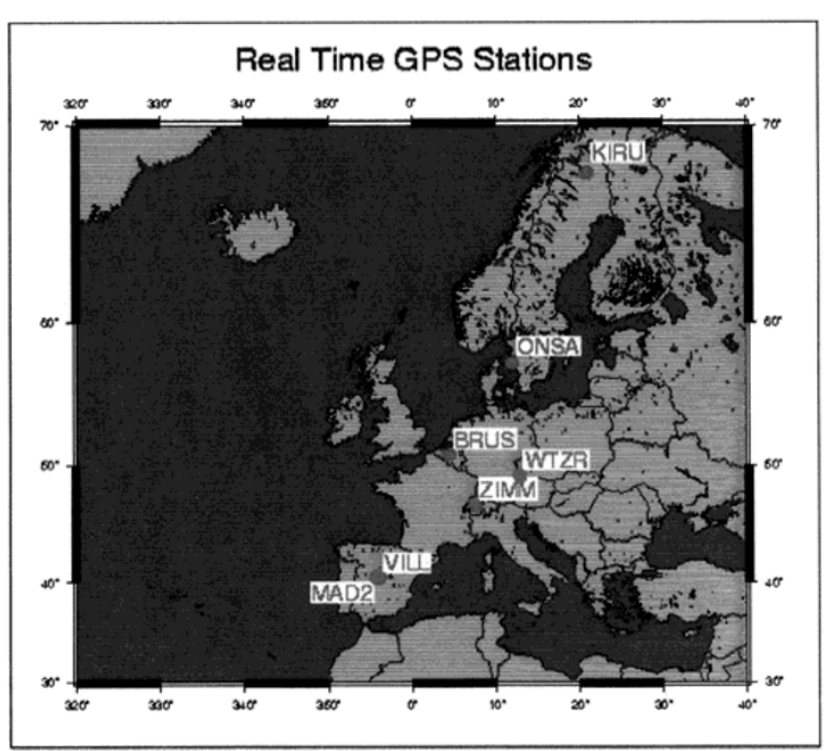

Fig. 2. Location of stations for the on-line system.

able within one hour after the files were closed and they were selected based on their availability and consistency of data. In Fig. 2 we show the real-time GPS stations location. For each station we collect a 1-hour batches of IGS data from the following servers: $\mathrm{BKG}^{4}$ (ftp://igs.ifag.de), $\mathrm{IGN}^{5}$ (ftp://igs.ensg.ign.fr), and CDDIS ${ }^{6}$ (ftp:// cddis.nasa.gov). The processing algorithm and the data quality checks are based on the off-line system procedure. Our goal is to provide a set of results within the next hour after data acquisition. The on-line products or results are: a) Zenith Total Delay (ZTD) monitored by each station, and b) Obtention of clock corrections for GPS satellites to be used in point positioning strategies. These results are available in the web site: http: / / www. ieec.fcr.es/gps / intro.html. This site is updated automatically for each 1-batch hour results. The ZTD time-series obtained with the real-time system are given in a format ready for codification in BUFR format ${ }^{7}$, so that the data can be distributed to meteorological institutions. The results are provided together with a formal variance value which provide an estimation of the accuracy. In order to prove further such accuracy we have performed some comparisons as shown below. However, the final validation of such data would come from their regular ingestion into meteorological models and provide an estimation of the impact of such data. We foresee an initial off-line system, similar to the one above described that would help in adjusting the parameters so that results are useful for weather predictions.

\subsection{Processing approach}

The first step is to collect hourly data from each GPS receiver included in the on-line network. We merge the 23 previous hours with the new file of 1 hour data, so our set of data is a "sliding window" of 24 hours. The use of such

\footnotetext{
${ }^{4}$ Bundesamt für Kartographie und Geodäsie.

${ }^{5}$ Institut GÈographique National.

${ }^{6}$ Crustal Dynamics Data Information System, NASA-GSFC.

${ }^{7}$ Binary Universal Format for the Representation of meteorological data, is the World Meteorological Organization standard for point data.
}

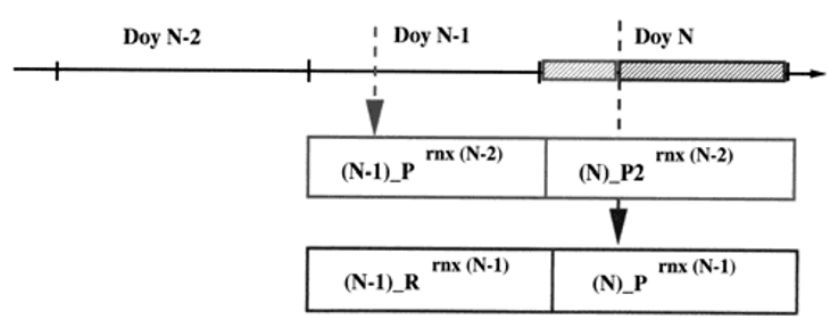

Fig. 3. Diagram of Orbit Predictions availability for different times of the day. Arrows indicate approximate time of issuing by the CODE provider.

sliding window provides the solution with a better accuracy because the stochastic process modeling the ZTD evolution benefits from a history of data of 24 hours instead of only one hour. In such an approach, we have to take care of the day boundary problem, particularly regarding the orbits and for that, orbits defined for different natural days are smoothed in the day boundary so that they are connected and there is no jump in the satellites orbit estimation. It is worth mentioning, also, that in such a system we are always considering the last batch of the solution and, hence, we will have the statistically worse part of the solution, since the Kalman filtering does not benefit from the smoothing process (see e.g. Herring et al. (1990) for a discussion on the smoothing process). The orbits for data processing are taken from the CODE center in the University of Berne (ftp: / / ubeclu. unibe.ch). The orbit predictions available are: Rapid prediction orbits (R orbits), 24 hours prediction orbits (P orbits), 48 hours prediction orbits ( $\mathrm{P} 2$ orbits), and 72 hours prediction orbits. For a good performance of our system we use the best available orbits at every time: P and P2 orbits for the first part of the day and $\mathrm{R}$ and $\mathrm{P}$ orbits as soon as they are available. In Fig. 3 we show a diagram of the availability of the orbits and how they are combined to cover the 24 hours of the sliding window of data. The Earth Rotation Parameters are estimated from IERS ${ }^{8}$ Bulletin B values, kept up to date from the server ftp: //iersb.obn.fr.

\subsection{Validation}

As with the off-line system, we need to validate the solutions using some reference values. Following the same approach we have taken the ZTD solutions using precise orbits and clock corrections provided by JPL and compared them with the 24 sets of the last one hour solutions provided by the on-line system; we selected one week period and computed the variance $(\sigma)$ and bias of the difference. Because we were using two sets of orbits ( $\mathrm{P}$ and P2, and R and P, as detailed above) we first considered the on-line system as if it always used the merging of P and P2 orbits and as if it always used $\mathrm{R}$ and $\mathrm{P}$, regardless of their availability (this analysis was obviously done a posteriori). We show the results in Table 3 and notice that there is little influence and that soultions have a subcentimeter accuracy although some $\mathrm{cm}$-level bias can be seen for some stations.

In order to verify the clock solutions provided in the on-line system, we have acted as with the off-line system and used them for a point positioning determination for five stations during one day and computed the accumulated bias and rms

\footnotetext{
${ }^{8}$ International Earth Rotation Service.
} 
Table 3. Comparison of solutions for two different combinations of orbits ( $\mathrm{P}$ and $\mathrm{P} 2$, and $\mathrm{R}$ and $\mathrm{P}$ ) with respect to the precise solution using final JPL products (bs stands for bias and units are in $\mathrm{cm}$ ).

\begin{tabular}{crrrr}
\hline STA & PP2 bs & PP2 $\sigma$ & RP bs & RP $\sigma$ \\
\hline BRUS & -1.063 & 0.156 & -0.828 & 0.156 \\
KIRU & -1.453 & 0.151 & -1.196 & 0.151 \\
ONSA & -0.417 & 0.162 & 0.023 & 0.163 \\
WTZR & 0.247 & 0.084 & 0.508 & 0.085 \\
ZIMM & -0.806 & 0.123 & -0.586 & 0.123 \\
\hline
\end{tabular}

Table 4. Comparison of the ZTD time series obtained using clock corrections from the on-line system and with JPL products. This show consistency of the solutions.

\begin{tabular}{ccc}
\hline STA & acc_bias $(\mathrm{cm})$ & acc_ $\sigma(\mathrm{cm})$ \\
\hline BRUS & 0.844 & 0.076 \\
KIRU & 0.319 & 0.066 \\
ONSA & 0.762 & 0.067 \\
WTZR & 0.668 & 0.065 \\
ZIMM & 1.097 & 0.102 \\
\hline
\end{tabular}

with respect to the solution using precise orbits and clocks. Results are presented in Table 4.

\section{Problems and Issues for the On-Line System}

Whereas for the validation and analysis of techniques offline systems have been used, the main point of the on-line system is to explore the design problems linked to the implementation of an exploitation system which must routinely operate. The main concerns of a near-real-time system are data availability and stations quality. As we show in Fig. 4, there is a correlation between the $\%$ of stations available for a particular hour data set and the error between the online time series solution and the precise solution. Since data availability can be ensured by the organization responsible of the stations, data transmission connections are therefore the issue. The mean data collection time from european servers is less than 10 minutes. This time increases for the US servers being up to 15 minutes, and sometimes is not at all possible because we use Internet networks for the acquisition, not dedicated lines. The data processing time is, in both cases, approximately 5 minutes on a Sun Ultra Sparc with 512 Mbytes RAM. The acquisition time is bottleneck for near-real-time processing since a minimum number of stations are needed and their availability can slow down the system. A set of back up stations must be designed to ensure this minimum requirement. An important limitation in the on-line system is the disk storage space: the results are kept for 24 hours and then removed in order not to collapse the available space in our computer system.
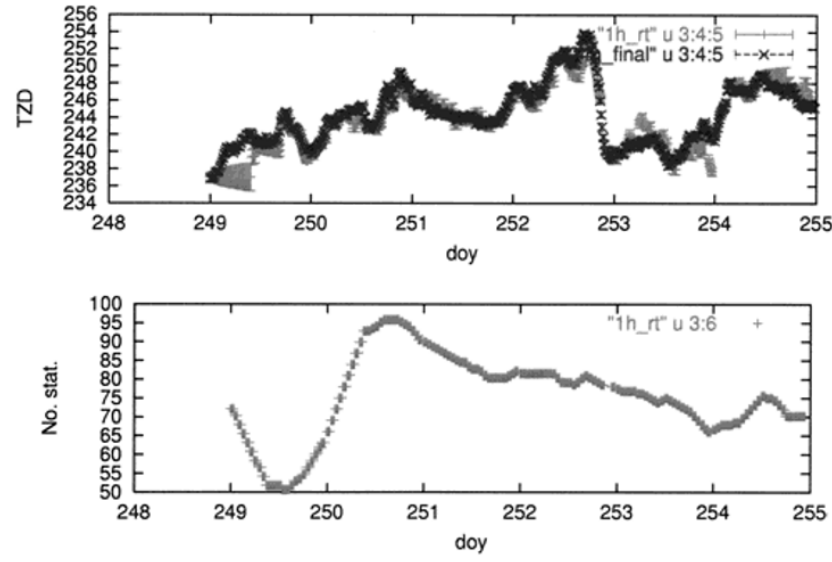

Fig. 4. Top: In dark grey, solution with Final Precise Orbits and in light grey, solutions obtained in the on-line system for the IGS station Onsala. Bottom: Percentage of available stations.

\section{Conclusions}

We have set up a system for a near-real-time processing of GPS data from an european network of receivers. IGS hourly data are obtained from public servers and are processed using the GIPSY-OASIS software and orbit predictions from CODE. Results are verified acquiring results using precise orbits and clock corrections, obtainig sub-centimeter accuracies for ZTD. Issues for the on-line system are identified, namely, orbit prediction quality, data availability and storage capability.

Acknowledgments. This work has been been supported by the CLIMAP (ENV4-CT97-0387) and MAGIC (ENV4-CT98-0745) projects and IGS organization.

\section{References}

Bevis, M., S. Businger, T. A. Herring, C. Rocken, R. A. Anthes, and R. Ware, GPS meteorology: Remote sensing of atmospheric water vapor using the global positioning system, J. Geophys. Res., 97(D14), 1578715801, 1992.

Duan, J., M. Bevis, P. Fang, Y. Bock, S. Chiswell, S. Businger, C. Rocken, F. Solheim, T. van Hove, R. Ware, S. McClusky, T. Herring, and R. King, GPS meteorology: Direct estimation of the absolute value of precipitable water, J. Appl. M., 24(24), 830-838, 1996.

Elgered, G., J. L. Davis, T. A. Herring, and I. I. Shapiro, Geodesy by radio interferometry: water vapour radiometry for estimation of the wet delay, J. Geophys. Res., 96(B4), 6541-6555, 1991.

Herring, T. A., J. L. Davis, and I. I. Shapiro, Geodesy by radio interferometry: the application of Kalman filtering to the analysis of Very Long Baseline Interferometry data, J. Geophys. Res., 95(B8), 12561-12581, August 1990.

Niell, A. E., Global mapping functions for the atmosphere delay at radio wavelengths, J. Geophys. Res., 101(B2), 3227-3246, 1996.

Webb, F. H. and J. F. Zumberge, An Introduction to GIPSY-OASIS II, Jet Propulsion Laboratory, California Institute of Technology, 1997.

Zumberge, M., M. B. Heflin, D. C. Jefferson, M. M. Watkins, and F. H. Webb, Precise point positioning for the efficient and robust analysis of GPS data from large networks, J. Geophys. Res., 102(B3), 5005-5017, 1997.

A. Flores, A. Escudero (e-mail: escudero@ieec.fcr.es), M. J. Sedó, and A. Rius 